International Journal of Engineering \& Technology, 6 (2) (2017) 29-35
International Journal of Engineering \& Technology
SPC
Website: www.sciencepubco.com/index.php/IJET
doi: $10.14419 /$ ijet.v6i2.7488
Research paper

\title{
A new approach using hybrid power series - cuckoo search optimization algorithm to solve electrostatic pull-in instability and deflection of nano cantilever switches subject to van der waals attractions
}

\author{
Jalal Alsarraf *, Khaled Alawadhi, Abdulwahab Alnaqi, S.A.M. Swilem \\ Public Authority for Applied Education and Training (PAAET) College of Technological Studies (CTS) \\ Automotive and Marine Engineering Department, Shuwaikh, 70654 Kuwait \\ *Corresponding author E-mail: jm.alsarraf@paaet.edu.kw
}

\begin{abstract}
A hybrid Power Series (PS) and Cuckoo Search via L'evy Flights (CS) optimization algorithm (PS-CS) method is utilized to obtain a solution for the deflection and pull-in instability of a nano cantilever switch in the presence of the van der Waals attractions, electrostatic forces and fringing filed effects. In order to obtain a relation for deflection of the beam, a trial solution including adjustable coefficients, satisfying the boundary conditions of the governing, is proposed. The cuckoo search optimization algorithm is executed to find the adjustable parameters of the trial solution satisfying the governing equation of the nanobeam. The results are compared with the available results in the literature as well as numerical solution. The results indicate the remarkable accuracy of the present approach. The minimum initial gap and the critical free standing detachment length of the nano actuator that does not stick to the substrate due to the van der Waals attractions, as an important parameter in pull-in instability of the nano switches, is calculated. Utilizing the results of the PS-CS, the stress distribution inside the nano actuator is determined at the onset of the pull-in instability.
\end{abstract}

Keywords: Cantilever Nano Actuator; Pull-In Instability; Electromechanical Switches.

\section{Introduction}

Many of the micro and nano devises including, switches in some microchips, pressure sensors, chemical sensors, values and pumps, are constructed using suspended beams and plates [1-3]. A cantilever nano switch is a beam suspended over a substrate. Applying voltage difference between the beam and the substrate attracts the beam into the substrate and causes the deflection of the beam. As the voltage difference between the beam and substrate increases, the deflection of the beam into the substrate increases. An increase of the voltage beyond a critical voltage, pull-in instability voltage, results in instability of the beam. In this case, the suspended beam suddenly jumps and sticks to the substrate. In the nanoscale, the nanobeam is also subject to the intermolecular forces. When the separation space between the beam and substrate is less than the retardation length, typically below $20 \mathrm{~nm}$, the van der Waals force is significant and affects the deflection of the nanobeam [4].

The nanobeams mostly are utilized in arrays [5]. For instance, hundreds of thousands of nanobeams in arrays are utilized to construct a digital memory. Therefore, analysis of nano switches and obtaining analytical or semi analytical relations for deflection or pull-in instability of these actuators would significantly reduce the computational cost of future designs.

Generally there are two main approaches for analysis of nano switches. In a simple approach, the nanobeam is assumed as a mass and spring suspended over the substrate. The mass is subject to the uniform applied forces [6], [7]. In this approach, calculating the internal stresses and mechanical failure the nanobeam is not possible. In the second approach, the nano beam is considered as a distributed parameter model. The distributed parameter model leads to a nonlinear differential equation which could provide details of the beam deflection and stress distribution along the beam.

Lin and Zaho [7] utilized the lamped model to analyze the pull-in instability of micro actuators. They obtained analytical relations for pull-in instability of the actuators. Soroush et al. [3] utilized a distributed parameter model to examine the pull-in instability and deflection of nano actuators. They [3] utilized the Adomian decomposition method to obtain a solution for deflection and pull-in instability of nano actuators. The results show that the Adomian series are capable to obtain a solution for pull-in instability of the nano actuators; however, the accuracy of the results is not good. Hence, Noghrehabadi et al. [9] tried to increase the accuracy of the Adomian power series solution by using the Pade approximations. The Pade approximants increased the accuracy of the solution. However, Pade approximants transformed the simple power series solution to a rational form, and in some cases the denominator could take zero digits which in this case the results are not applicable. Ramezani et al. $[9,10]$ utilized the Green method to obtain a closed-form solution of the pull-in instability in nanocantilevers. They $[9,10]$ assumed a second order shape function for the shape of nanobeam. Recently, artificial intelligence techniques have been utilized to solve various types of differential equations [11-14]. Meade and Fernandez [11] and Lagaris, Likas [12] proposed the neural networks as a trial function with adjustable parameters for solving boundary value differential equations. Malek and Beidokhti [13] proposed a new approach for solving 
differential equations using a power series as a trial function with adjustable parameters and the Nelder-Mead optimization algorithm to adjust the parameters.

Cuckoo Search via L'evy Flights algorithm proposed by Yang and Deb [15] as a population base optimization algorithm. This algorithm mimics the obligate brood parasitism of some cuckoo species which lay their eggs in the nests of other Cuckoo species. The effectiveness of this algorithm has been demonstrated in many recent studies [16-18].

In the present study a combination of power series and Cuckoo search via Levy flights optimization algorithm is proposed as a new approach to obtain a relation for deflection of nano cantilever beams in the presence of van der Waals attractions. The pull-in instability, free standing length of the beam and the Stress resultants are also evaluated.

\section{Mathematical model}

A schematic view of a nano-cantilever beam suspended over a conductive substrate is depicted in Fig. 1.

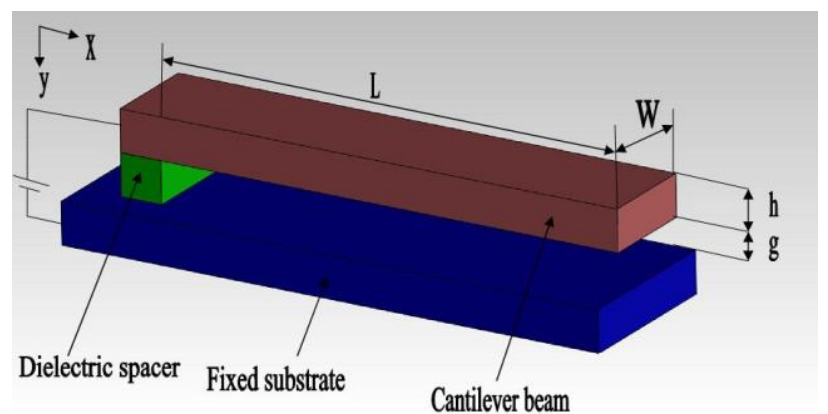

Fig. 1: Schematic View of the Physical Model of the Cantilever Nano Switch.

The cross section of the beam is rectangular with thickness of $h$ and width w. Length of the beam is denoted by L. The initial gap space between the cantilever beam and substrate plane is denoted by g. Considering the van der Waals attractions, electro static forces and fringling effects, the governing equation for the distributed model of the beam is written as [3]:

$\frac{d^{4} u}{d x^{4}}=\frac{\alpha}{(1-u(x))^{3}}+\frac{\beta}{(1-u(x))^{2}}+\frac{\gamma \beta}{(1-u(x))}$

Subject to the following boundary conditions at the basis and the natural end:

$$
\mathrm{u}(0)=\mathrm{u}^{\prime}(0)=0 \text {, at } \mathrm{x}=0 \text { and } \mathrm{u}^{\prime \prime}(1)=\mathrm{u}^{\prime \prime \prime}(1)=0, \quad \text { at } \mathrm{x}=1
$$

Where $\mathrm{x}$ is the non-dimensional position along the beam measured from the clamped end, and prime denotes the differentiation with respect to $\mathrm{x} . \alpha$ is the non-dimensional van der Waals parameter, $\beta$ is the non-dimensional electro static parameter and $\gamma$ is the nondimensional fringing filed parameter. The non-dimensional parameters are defined as follow [3]:

$\alpha=\frac{A w L^{4}}{6 \pi g^{4} E_{\text {eff }} I} \quad \beta=\frac{\varepsilon_{0} w^{2} L^{4}}{2 g^{3} E I} \quad \gamma=0.65 \frac{g}{w} \quad x=\frac{X}{L} \quad u=\frac{y}{g}$

Where $y$ is the beam deflection, $\varepsilon_{0}=8.854 \times 10^{-12} c^{2} N^{-1} m^{-2}$ is the permittivity of the vacuum, $\mathrm{V}$ is the applied external voltage $h=1.055 \times 10^{-34} J_{S}$ is Planck's constant divided by $2 \pi$, and $c=2.998 \times 10^{8} \mathrm{~m} / \mathrm{s}$ is the light speed. Eeff is the effective Young's modulus, and $\mathrm{I}$ is the moment of inertia of the beam cross section $\left(w^{3} / 12\right)[3]$.

\section{Cuckoo search l'evy flight}

Yang and Deb [15] proposed the Cuckoo Search L'evy flight (CS) method as a new meta-heuristic algorithm to deal with unconstrained optimization problems. The Cuckoo search L'evy flight algorithm is inspired by the obligate brood parasitic behavior of some cuckoo species in combination with the L'evy flight behavior of some birds and fruit flies in nature. CS method simply follows three rules as follows [15]:

1) Each cuckoo lays one egg at a time, and dump its egg in a randomly chosen nest; 2) The best nests with high quality of eggs will carry over to the next generations; 3 ) The number of available host nests is fixed, and the egg laid by a Cuckoo is discovered by the host bird with a probability $p_{a} \in\left[\begin{array}{ll}0 & 1\end{array}\right]$.

As the CS method tries to maximize a fitness function, in the case of minimization the fitness function is considered as the minus of the original fitness function. Each egg in a nest symbolizes a solution, and a cuckoo egg symbolizes a new candidate solution. The goal is to use the new and potentially better solutions (cuckoo egg) to replace a not so good solution in the nests.

Utilizing the mentioned three basic rules of CS method, the main steps of the CS algorithm are listed as the pseudo code in Fig. 2.

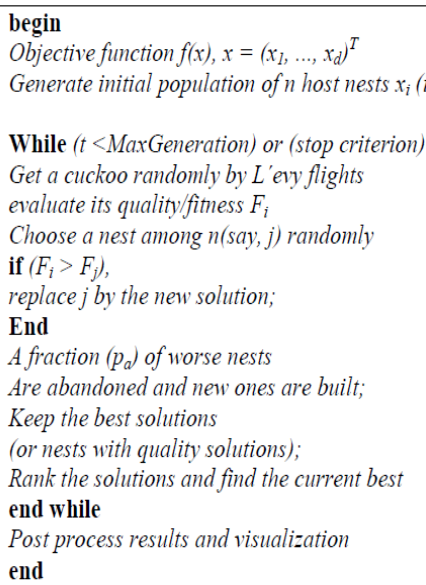

Fig. 2: Pseudo Code of the Cuckoo Search (CS) [15].

For generating a new solution (a new cuckoo egg i) for next generation $\mathrm{x}^{(\mathrm{t}+1)}$, a L'evy flight is required to be performed as follow (3) where $\kappa>0$ is the step size of the search, related to the optimization parameter scales of the optimization problem. The default use

$$
x_{i}^{(t+1)}=x_{i}^{(t)}+\kappa \otimes t^{-\lambda} \quad 1 \leq \lambda \leq 3 \otimes \quad \text { Of } \kappa=1
$$

Is a good choice in most cases. The product denotes the entry wise multiplication which is similar to those used in Particles Swarm Optimization (PSO) method [19, 20].

More details about the implementation of the cuckoo search l'evy flight method can be found in the work of Yang and Deb [15].

\section{Problem formulation}

The deflection of the beam subject to the external forces can be obtained from the solution of Eq. (1-a) subject to the boundary conditions of eq. (1-b). In order to find a solution for Eq. (1), consider a uniform discretization of the governing differential equation of Eq. (1-a) with $\mathrm{m}$ arbitrary points [12], [13]. In each discretized point the governing equation can be written as: 


$$
\frac{d^{4} u\left(x_{i}\right)}{d x^{4}}-\left(\begin{array}{l}
\frac{\alpha}{\left(1-u\left(x_{i}\right)\right)^{3}}+ \\
\frac{\beta}{\left(1-u\left(x_{i}\right)\right)^{2}}+ \\
\frac{\gamma \beta}{\left(1-u\left(x_{i}\right)\right)}
\end{array}\right)=0, x_{i} \in D, \quad i=1,2, \ldots, m
$$

Eq. (4) represents Eq. (1-a) is discretized points. Now, let's assume $\mathrm{y}_{\mathrm{T}}(\mathrm{x}, \stackrel{\mathrm{r}}{\mathrm{a}})$ as a function (a trial solution) which approximate the solution to Eq. (1). a denotes a vector containing the adjustable parameters. As the solution of Eq. (1) should satisfy the governing equation, the Eq. (4) should hold true for each point in the domain of the solution. Substituting the assumed trial solution in Eq. (4) should also satisfy the equation in each discrete point of the domain of the solution. The error of the trial solution in the domain of the solution will be computed using the following equation:

$$
E(\stackrel{r}{a})=\sum_{i=1}^{m}\left(\frac{d^{4} y_{T}\left(x_{i}, a\right)}{d x^{4}}-\left(\frac{\frac{r}{\left(1-y_{T}\left(x_{i}, a\right)\right)^{3}}}{\frac{\beta}{\left(1-y_{T}\left(x_{i}, a\right)\right)^{r}}}+\right)\right)^{2}, x_{i} \in\left[\begin{array}{ll}
0 & 1
\end{array}\right]
$$

The value of $E(\stackrel{a}{a})$ in Eq. 5 should be zero if the trial solution satisfies the governing differential equation, Eq. (1). However, as $\mathrm{y}_{\mathrm{T}}(\mathrm{x}, \mathrm{r})$ is the approximate solution of Eq. (1), the value of $E(\stackrel{a}{a})$ is not zero. The boundary conditions of the governing equation should also be satisfied. Therefore, the trial solution is written as the sum of two parts, the first part will satisfy the boundary conditions exactly. The second part with the aid of the first part constructs a trial solution for Eq. (1) as follow:

$$
\begin{aligned}
& Y_{T}(x, \stackrel{r}{a})=a_{1} x^{5}+a_{2} x^{4}-\left(10 a_{1}-4 a_{2}\right) x^{3} \\
& +\left(20 a_{1}+6 a_{2}\right) x^{2}+x^{2}(x-1)^{4} N(x, a)
\end{aligned}
$$

Where the coefficients of $\mathrm{a}_{1}$ and $\mathrm{a}_{2}$ as well as the vector of $\stackrel{\mathfrak{a}}{a}$ are the adjustable parameters. The term of $N(x, a)$ is a power series in the form of $\left(N(x, a)=\sum_{i=0}^{n} a_{i+3} x^{i}\right)$ involving the remaining adjustable parameters (i.e. $a_{3} \ldots a_{n}$ ). It should be noticed that the trail function in the form of Eq. (6) exactly satisfies the boundary conditions of Eq. (1-b). Now, the adjustable parameters of $\mathrm{a}_{1}, \mathrm{a}_{2}$ and $\stackrel{1}{a}$ should be evaluated such a way that $E(a)$ in Eq. (5) be minimum. Here, the Cuckoo search l'evy flight is utilized to find the values of a and $\mathrm{a}_{2}$ and the vector $\stackrel{1}{a}$ regarding to minimize $E(a)$ in Eq. (6). The Cuckoo search l'evy flight optimization algorithm is coded using MATLAB 2009 program. The calculations were also executed using the MATLAB Software.

\section{Results}

21 sample points $(\mathrm{m}=21)$ with uniform space of 0.5 were chosen in the domain of the solution (xi $€\{0,0.05,0.01 \ldots 1\}$ ). In the Cuckoo search l'evy flight optimization algorithm the number of the nests (nests) and the fraction of worse nests $\left(\mathrm{P}_{\mathrm{a}}\right)$ were fixed at 15 and 0.25 respectively. 300 iterations were also selected for generations number (Number of Iterations).

As a test of the convergence of the solution, the deflection of a typical nano cantilever actuator $(\alpha=0.3, \beta=0.2, \mathrm{~g} / \mathrm{w}=1.0)$ is computed. In this case, the results of present study are compared with the numerical results as well as the results of Adomian decomposition method [3]. The numerical results are obtained using the dsolve function of MAPLE 14.0 mathematical software [21], [22]. Table 1 shows the variation of the cantilever tip deflection $\left(\mathrm{u}_{\mathrm{tip}}\right)$ as a function of the dimensionless length of the beam (x) for different selected terms of the size of the trial solution. The error in Table 1 denotes the difference between the tip deflection (evaluated using the Adomian method [3] or the present solution) and that of the numerical solution. Table 1 shows that the trial solution with six terms of the power series or more provides excellence results.

\begin{tabular}{|c|c|c|c|}
\hline \multicolumn{2}{|c|}{ Method } & $\begin{array}{c}\text { Adomian } \\
{[3]}\end{array}$ & $\begin{array}{l}\text { Present meth- } \\
\text { od }\end{array}$ \\
\hline \multicolumn{2}{|c|}{ Numerical } & \multicolumn{2}{|c|}{0.09081} \\
\hline \multirow{2}{*}{$\begin{array}{c}4 \text { Terms } \\
\text { series }\end{array}$} & $\mathrm{u}_{\text {tip }}$ & 0.0788 & 0.085100 \\
\hline & Errors & 0.0121 & 0.005698 \\
\hline \multirow{2}{*}{$\begin{array}{c}6 \text { Terms } \\
\text { series }\end{array}$} & utip & 0.0986 & 0.090810 \\
\hline & Errors & 0.0077 & $4.03 \mathrm{E}-05$ \\
\hline \multirow{2}{*}{$\begin{array}{c}7 \text { Terms } \\
\text { series }\end{array}$} & $\mathrm{u}_{\text {tip }}$ & 0.0870 & 0.090811 \\
\hline & Errors & 0.0038 & 3.07E-05 \\
\hline \multirow{2}{*}{$\begin{array}{l}8 \text { Terms } \\
\text { series }\end{array}$} & $\mathrm{u}_{\text {tip }}$ & 0.0940 & 0.090808 \\
\hline & Errors & 0.0032 & $2.06 \mathrm{E}-06$ \\
\hline \multirow{2}{*}{$\begin{array}{l}9 \text { Terms } \\
\text { series }\end{array}$} & $\mathrm{u}_{\text {tip }}$ & 0.0886 & 0.090841 \\
\hline & Errors & 0.0022 & $4.86 \mathrm{E}-07$ \\
\hline \multirow{2}{*}{$\begin{array}{c}10 \\
\text { Terms } \\
\text { series }\end{array}$} & $\mathrm{u}_{\text {tip }}$ & 0.0922 & 0.090770 \\
\hline & Errors & 0.0014 & $1.50 \mathrm{E}-08$ \\
\hline
\end{tabular}

Table 1: The Variation of the Tip Deflection of A Typical Beam with Respect to X Obtained Using Different Selected Terms of Power Series for $\mathrm{A}=0.3, \mathrm{~B}=0.3$, and $\mathrm{G} / \mathrm{W}=1$ :

It is also clear that as the size of the power series in the trial solution rises the accuracy of the solution would also increases. Table 1 also shows that eight terms of the power series in the trial solution provides results with error of the order of $10^{-6}$, but the Adomian series solution with eight terms of the series solution provides results with error of the order of $10^{-3}$. Therefore, it is clear that the present method with the same size of the series could provide very accurate results. As the eight terms in the trial solution provide excellence accuracy, the results in the rest of the paper are evaluated using eight terms of the power series for convenience.

The corresponding trial power series and the power series obtained by Adomian method [3] for the results of Table 1 (i.e. $\alpha=0.3, \beta=0.2$ and $g / w=1$ ) can be summarize as follows respectively:

$u(x)=0.178337302 x^{2}-0.113517738 x^{3}$

$+0.024877493 \mathrm{x}^{4}+0.000028350 \mathrm{x}^{5}+$

$0.002011236 \mathrm{x}^{6}-0.001214524 \mathrm{x}^{7}+0.000285856 \mathrm{x}^{8}$

$u(x)=0.183945211 x^{2}-0.116761146 x^{3}+$

$0.026250000 x^{4}-0.000730671253 x^{6}-$

$0.000198771951 x^{7}+0.0000732989145 x^{8}$ 
Indeed, Eqs. (7) and (8) represent the non-dimensional shape of the cantilever beam after deflection.

\subsection{Pull-in instability study}

The pull-in stability of the cantilever nano switch can be evaluated using the solution of Eq. (1) by setting $\mathrm{du}(1) / \mathrm{d} \xi \rightarrow \infty$ where $\xi$ is the parameter of study (i.e. $\alpha, \beta$ or $g / w$ ). When the nano switch reaches to the pull-in instability any increase in the magnitude of $\xi$ would results in large deflection of the beam (i.e. large variation of tip displacement). In the pull-in condition, the values of $\alpha, \beta$ or $\mathrm{g} / \mathrm{w}$ are denoted by subscript of PI. Any raise of $\xi$ to the values larger than the pull-in value, results in instability of the beam; in this case the governing equation cannot provide any real solution for deflection of the beam. In a case in which there is not any applied voltage between the electrode and the substrate, the suspended beam is solely subject to the intermolecular force. If the van der Waals attraction be large enough to induce pull-in instability the nano actuator will switch without any applied external voltage. In this situation the non-dimensional value of van der Waals parameter can be obtained by setting $\beta=0$ and du (1)/d $\alpha \rightarrow \infty$.

\subsubsection{Free standing parameters}

As mentioned in the previous section, when the separation space (g) between the substrate and the suspended cantilever beam is small enough, the movable beam might collapse onto the substrate because of the van der Waals attraction forces without applying any external voltage. Fig. 3 depicts the variation of nondimensional tip of the cantilever beam $\left(\mathrm{u}_{\mathrm{tip}}\right)$ as a function of the van der Waals parameter $(\alpha)$ when the applied external voltage difference is zero $(\beta=0)$.

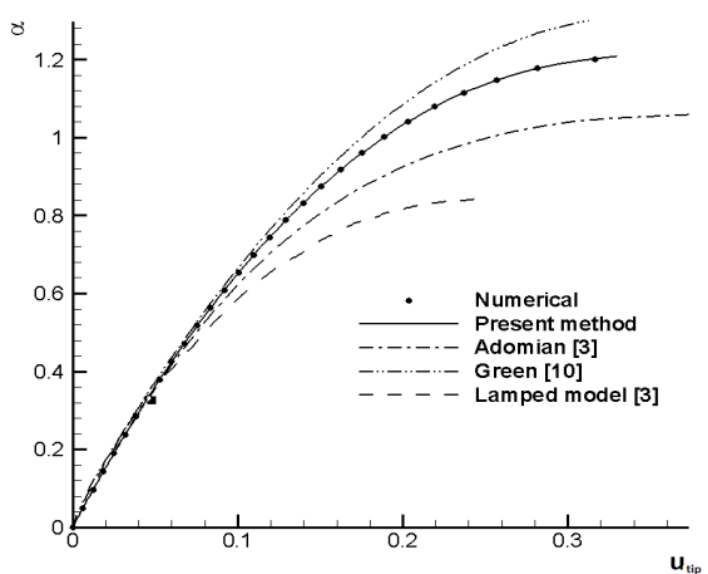

Fig. 3: Tip Deflection as A Function of Van Der Waals Parameter A.

As seen, the increase of van der Waals parameter increases the tip deflection of the beam until at a critical value of the van der Waals parameter, $\alpha_{\mathrm{C}}=1.21$, the pull-in instability occurs. In this case, the shape of the beam using PS-CS is obtained as follow:

$$
u(x)=0.607231800 x^{2}-0.298579773 x^{3}+
$$

$0.001136843 \mathrm{x}^{4}-0.001377942 \mathrm{x}^{5}+0.058577877 \mathrm{x}^{6}-$

\section{$0.043236974 \mathrm{x}^{7}+0.011599078 \mathrm{x}^{8}$}

A comparison between the results of present study (PS-CS method) and the results of previous studies is performed in Fig. 3. This figure shows that the accuracy of present PS-CS method is excellence compared with the numerical method.

For a nano switch with a specified value of the separation space (g) and in the absence of any external voltage, the maximum free standing length of the actuator, in which the nano actuator does not go through the pull-in instability, is called the detachment length $\left(\mathrm{L}_{\max }\right)$ [10]. Alternatively, for a specified cantilever nano switch with an specified value of the length (L), there is a minimum separation space, $\left(g_{\min }\right)$, in which the cantilever beam would not stick to the substrate because of the van der Waals attraction forces [10]. The detachment length $\left(\mathrm{L}_{\max }\right)$ and minimum separation space $\left(\mathrm{g}_{\min }\right)$ of the cantilever actuator are the basic parameters for design of nano electro mechanical switches. These parameters can be evaluated by using the critical value of van der Waals parameter, i.e. $\alpha$ c. Substituting the critical value of the van der Waals parameter $\left(\alpha_{\mathrm{C}}=1.21\right)$ into the definition of van der Waals parameter (i.e. $\alpha$ in Eq. 2); the relations for evaluation of $L_{\max }$ and $g_{\min }$ are obtained as follow:

$\mathrm{L}_{\max }=\left(0.605 \frac{\pi \mathrm{g}^{4} \mathrm{E}_{\mathrm{eff}} \mathrm{h}^{2}}{\mathrm{w}}\right)^{\frac{1}{4}} \mathrm{~g}_{\min }=\left(\frac{\mathrm{L}_{\mathrm{w}}}{0.605 \pi \mathrm{E}_{\text {eff }} \mathrm{h}^{2}}\right)^{\frac{1}{4}}$

\subsubsection{Electrostatic force}

Figs. (4) and (5) show the tip deflection of a wide $(\mathrm{g} / \mathrm{w}=0)$ and narrow $(\mathrm{g} / \mathrm{w}=1)$ cantilever beam as a function of electro static parameter, respectively, when the van der Waals attractions are negligible $(\alpha=0)$. Figs. (4) and (5) show that the tip deflection of a narrow beam is larger than the tip deflection of a wide beam. A comparison between the results of PS-CS and previous results is shown in Figs. (4) and (5). The results show that the lamped parameter model cannot properly follow the variation of tip deflection accurately. The lamped parameter model under estimated the tip deflection while the Green method overestimated the tip deflection of the nano beam.

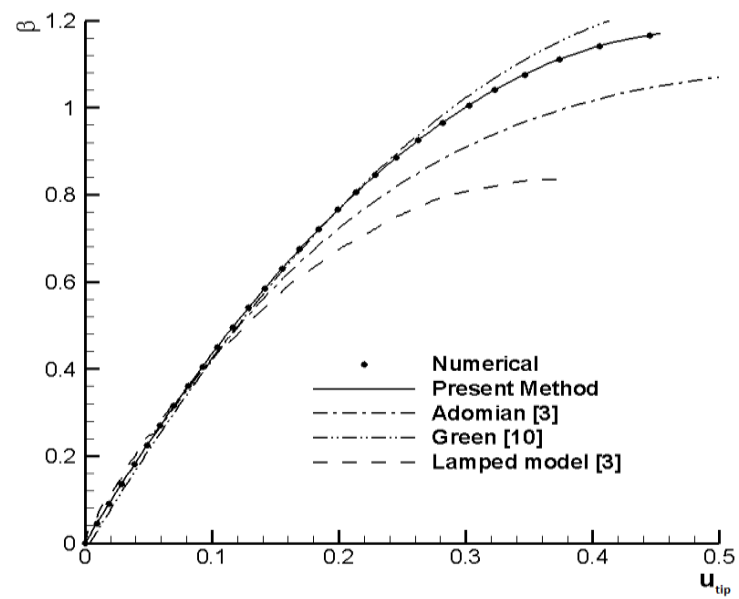

Fig. 4: Tip Deflection as A Function of Electrostatic Force (B) when $A=0$ and $\mathrm{G} / \mathrm{W}=0$.

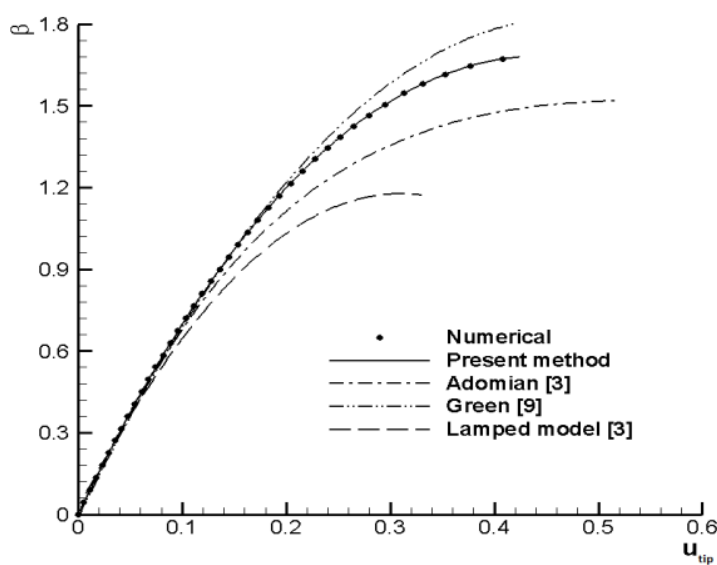

Fig. 5: Tip Deflection as A Function of the Electrostatic Parameter (B) when $\mathrm{A}$ and $\mathrm{G} / \mathrm{W}=1$. 
Figs (6) and (7) show the effect of fringing filed (for different values of $\mathrm{g} / \mathrm{w}$ parameter) on the pull-in values of elector static parameter $(\beta \mathrm{PI})$ and the corresponding tip deflection of the nano beam upI respectively, when $\alpha=0$.

Fig. (6) demonstrates that the Lamped parameter method [3] as well as the Adomian method [3] underestimated the pull-in value of the electro static parameter $\left(\beta_{\mathrm{PI}}\right)$ while the Greens method [10] overestimated magnitude of $\beta$ PI. Fig. (7) shows that the Green method [10] and the Adomian method [3] overestimated the tip deflection of the beam at the pull-in instability, and the lamped parameter method [3] under estimated the pull-in tip deflection of the beam. Figs. (6) and (7) show that the evaluated pull-in values of $\beta$ PI and UPI are in very good agreement with the numerical results.

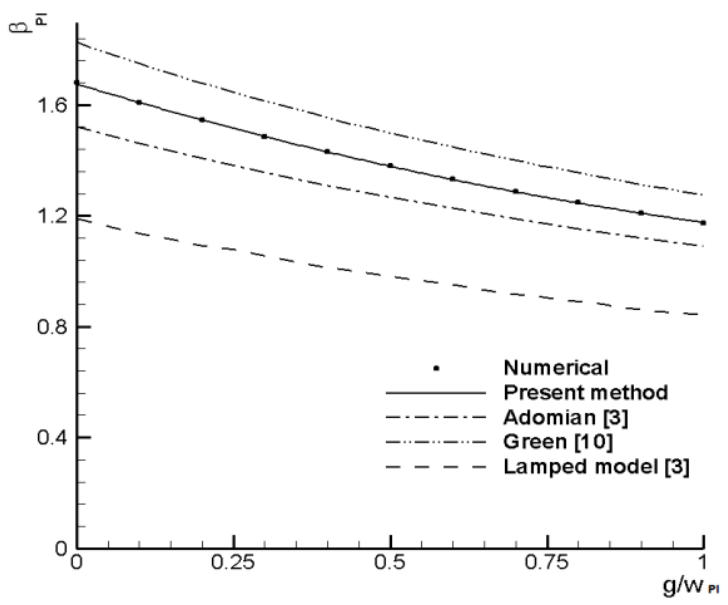

Fig. 6: Effect of Fringing Filed on B Pull-In.

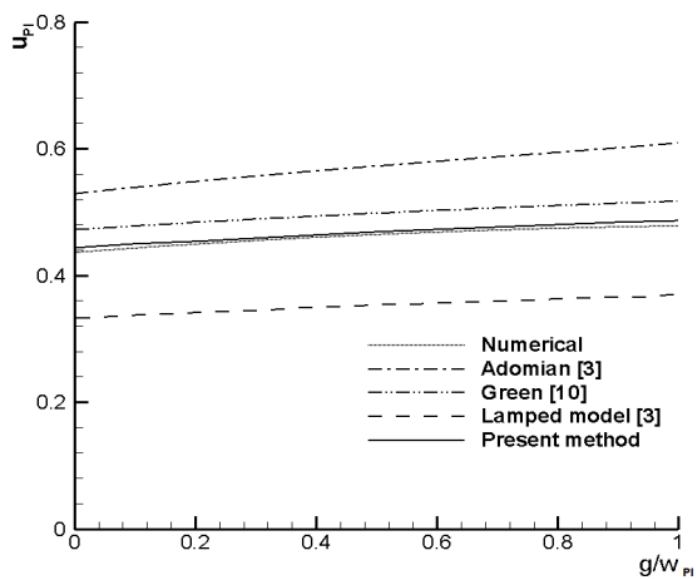

Fig. 7: Effect of Fringing Filed on $U_{p i}$ Pull-In.

\subsubsection{Electrostatic and intermolecular force at nanoscale sep- arations}

Fig. (8) shows the deflection of a cantilever nano-beam subject to simultaneous effects of electrostatic and van der Waals forces. This figure shows that when the applied external voltage is zero $(\beta=0)$ the shape of the beam is not at rest, and There is an initial deflection is because of the van der Waals forces $(\alpha=0.5)$. A comparison between the evaluated shape of the beam using the numerical method, eight terms of Adomian series [3] and eight terms of PS-CS method is performed in Fig. 8. As seen, the difference between Adomian results and the numerical solution is significant at the onset of pull-in instability. The results of PS-CS method indicate that the pull-in occurs at $\beta_{\mathrm{PI}}=0.66$ when $\alpha=0.5$ and $\mathrm{g} / \mathrm{w}=1$. The corresponding power series is obtained as follow: $u(x)=0.742382895 x^{2}-0.371064051 x^{3}+$

$0.006908911 x^{4}-0.002064628 x^{5}+0.070527153 x^{6}$

$-0.052763152 x^{7}+0.014290035 x^{8}$

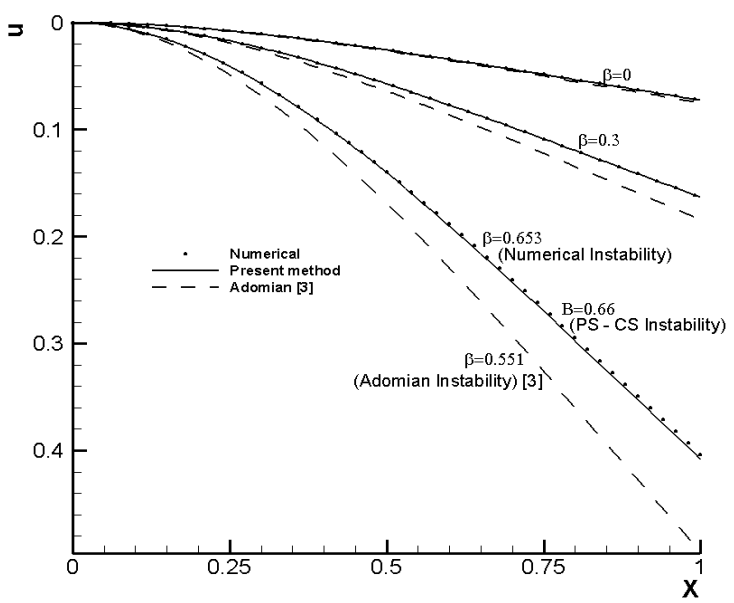

Fig. 8: Deflection of A Typical Nano-Beam Under Both Electrostatic and Intermolecular Loading when B Increases from Zero tTo Instability Point. $\mathrm{A}=0.5$ and $\mathrm{G} / \mathrm{W}=1$, Collapse Occurs when B Reaches Values Greater Than Its Critical Value $\mathrm{B}=0.66$

Fig. (9) Shows the pull-in value of the electro static parameter $\left(\beta_{\mathrm{PI}}\right)$ as a function of van der Waals parameter $\left(\alpha_{\mathrm{PI}}\right)$ for the narrow $(\mathrm{g} / \mathrm{w}=1)$ and wide $(\mathrm{g} / \mathrm{w}=0)$ beams. This figure depicts that as the intermolecular force $(\alpha)$ increases the required applied voltage $\left(\beta_{\mathrm{PI}}\right)$ in which the pull-in instability occurs decreases. The variation of electro static parameter as a function of the van der Waals parameter is almost linear. The pull-in tip deflection of the beam as a function of the van der Waals parameter is plotted in Fig. (10). This figure shows that as the van der Waals forces increases the tip deflection of the beam increases. A comparison between the results of PS-CS, numerical results as well as Adomian [3] and lamped model [3] is performed in Figs. (9) and (10). As seen the results of PS-CS are in very good agreement with the numerical results.

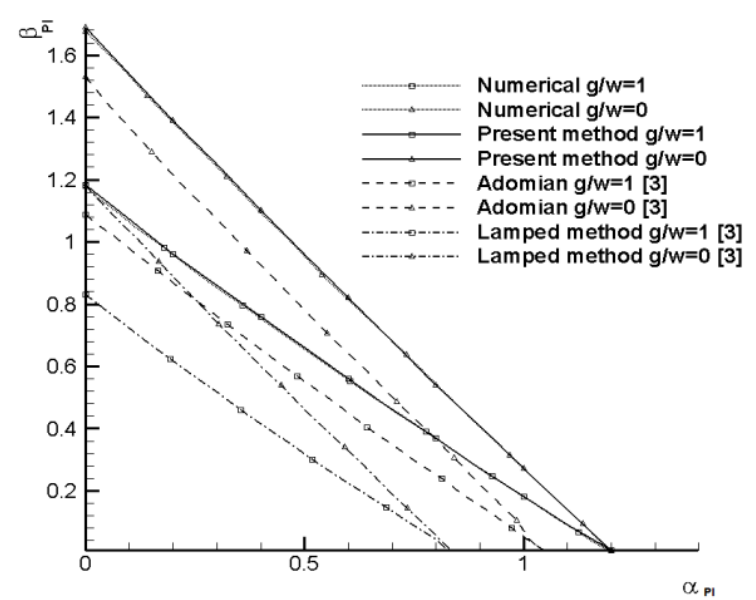

Fig. 9: Relationship between A and B Pull-In for Narrow and Wide Beams. 


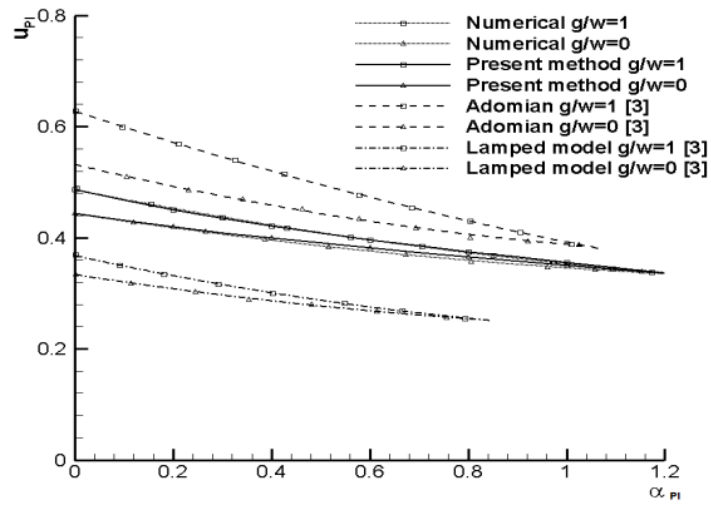

Fig. 10: Effect of Intermolecular Force on the $\mathrm{U}_{\mathrm{pi}}$ Pull-In.

\subsubsection{Stress resultants}

The stress distribution in the nanobeam is one of the basic requirements in the nano switches designs. The maximum value of the shear stress at the onset of the pull-in instability is very important because of its crucial effect on the failure of the switch. Utilizing the Euler beam theory, the shear stresses can be directly evaluated using the stress resultants [23]. In order to determine the critical values of stress resultants, $\mathrm{FPI}_{\mathrm{PI} \text { max }}$ and $\mathrm{M}_{\mathrm{PI} \text {,max }}$ are introduced as the dimensionless maximum value of the shear force and bending moment at the onset of instability, respectively as follow

$$
F_{P I, \text { max }}=\frac{F_{0} L^{3}}{E_{\text {eff }} \text { Ig }}, \quad M_{P I, \text { max }}=\frac{M_{0} L^{2}}{E_{\text {eff }} \text { Ig }}
$$

Where $\mathrm{F}_{0}$ and $\mathrm{M}_{0}$ are the shear force and the bending moment at the cross-section of the beam fixed end $(x=0)$. By these definitions, MPI,max and FPI,max equal to $\mathrm{u}^{\prime \prime}(\mathrm{x}=0)$ and $-\mathrm{u}^{\prime \prime \prime}(\mathrm{x}=0)$, respectively [23].

$\mathrm{F}_{\mathrm{PI} \text {,max }}$ and MPI,max as a function of the van der Waals force for wide and narrow types of cantilever nanobeams are depicted in Figs. (11) and (12), respectively. As seen, the increase of the van der Waals parameter ( $\left.\alpha_{\mathrm{PI}}\right)$ increases the maximum value of the shear forces. Therefore, the nano switches with smaller separation spaces (large values of $\alpha_{\mathrm{PI}}$ ) are subject to larger shear stresses. In addition, a comparison between the previous reported results and the results of present study is performed in Figs. (11) and (12). These figures show good agreement between the results of PS-CS and numerical results.

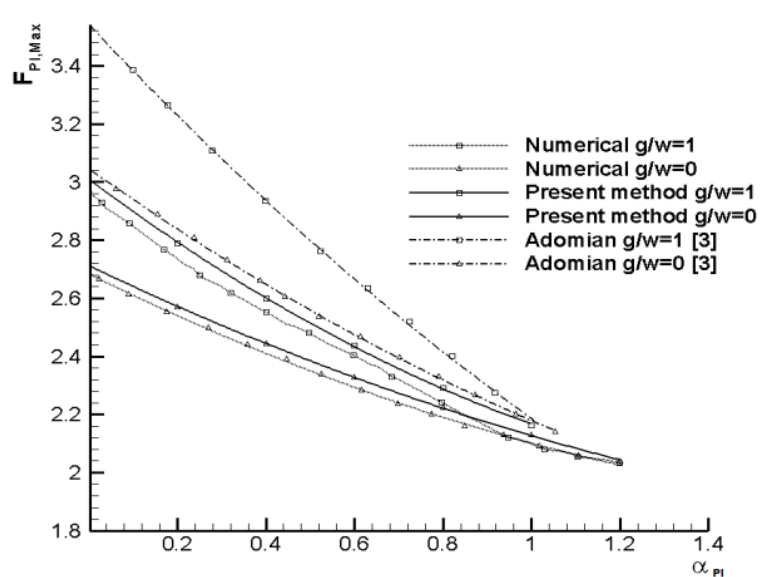

Fig. 11: Effect of Intermolecular Force on the Shear Force of Nano Cantilevers for Narrow and Wide Beams.

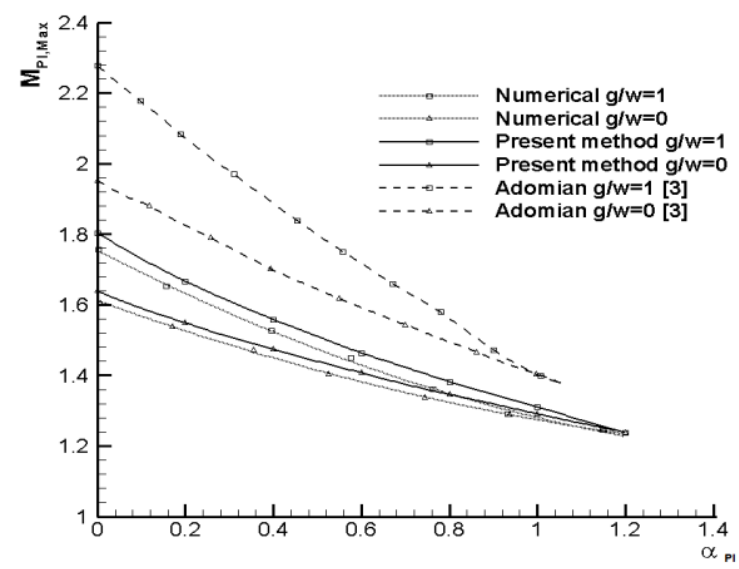

Fig. 12: Effect of Intermolecular Force on the Bending Moment of Nano Cantilevers for Narrow and Wide Beams.

\section{Conclusions}

A combination of power series as a trial solution and cuckoo search optimization algorithm is successfully utilized to obtain a relation for the shape of cantilever nano switches in the presence of van der Waals attractions and electro static forces. The pull-in instability of the nano switch is also studied. The results of present approach are compared with the numerical results as well as the reported results of Adomian, Green and Lamped methods in the literature. The comparison between numerical solution and the present approach demonstrates that the PS-CS approach overcomes the shortcoming of the accuracy of the previous approaches, and it is capable to deal with the problem robustly. Utilizing the obtained results, a relation for the detachment length and minimum separation space for nano cantilever switches is obtained. Finally, the stresses at the onset of pull-in instability are evaluated.

\section{References}

[1] Sinha, N., Wabiszewski, G. E., Mahameed, R., Felmetsger, V. V., Tanner, S. M., Carpick, R. W., \& Piazza, G. (2009, June). Ultra thin AlN piezoelectric nano-actuators. In Solid-State Sensors, Actuators and Microsystems Conference, 2009. TRANSDUCERS 2009. International (pp. 469-472). https://doi.org/10.1109/sensor.2009.5285460.

[2] Cui, J. B., Sordan, R., Burghard, M., \& Kern, K. (2002). Carbon nanotube memory devices of high charge storage stability. Applied

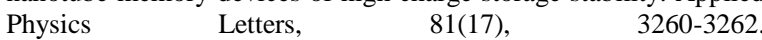
https://doi.org/10.1063/1.1516633.

[3] Soroush A, Koochi A, Kazemi AS, Noghrehabadi A, Haddadpour $\mathrm{H}$, Abadyan M. Investigating the effect of Casimir and van der Waals attractions on the electrostatic pull-in instability of nano$\begin{array}{llllll}\text { actuators. J. Phys. Scr. 2010; } 82 & 045801 .\end{array}$ https://doi.org/10.1088/0031-8949/82/04/045801.

[4] Mastrangelo $\mathrm{CH}, \mathrm{CH} \mathrm{Hsu}, 1993$ Mechanical stability and adhesion of microstructures under capillary force-Part I: Basic theory Journal $\begin{array}{llll}\text { of } & \text { Microelectromechanical } & \text { Systems } 2 & \text { (1): 33-43 }\end{array}$ https://doi.org/10.1109/84.232593.

[5] Lieber, C. M., Rueckes, T., Joselevich, E., Kim, K. (2001). Nanoscopic wire-based devices, arrays, and methods of their manufacture, Google Patents.

[6] Wen-Hui Lin, and Ya-Pu Zhao, Pull-in Instability of Micro-switch Actuators: Model Review, International Journal of Nonlinear Sciences and Numerical Simulation, 9(2), 175-183, 2008 https://doi.org/10.1515/IJNSNS.2008.9.2.175.

[7] Lin, W. H., \& Zhao, Y. P. (2005). Casimir effect on the pull-in parameters of nanometer switches. Microsystem Technologies, 11(23), 80-85. https://doi.org/10.1007/s00542-004-0411-6.

[8] Noghrehabadi, A., Ghalambaz, M., \& Ghanbarzadeh, A. (2012). A new approach to the electrostatic pull-in instability of nanocantilever actuators using the ADM-Padé technique. Computers \& Mathematics with Applications, 64(9), 2806-2815. https://doi.org/10.1016/j.camwa.2012.04.013.

[9] Ramezani A, Alasty A, Akbari J., Closed-form approximation and numerical validation of the influence of van der Waals force on 
electrostatic cantilevers at nano-scale separations. Nanotechnology, 2008; 19015501 (10pp).

[10] Ramezani A, Alasty A, Akbari J., Closed-form solutions of the pull-in instability in nano-cantilevers under electrostatic and intermolecular surface forces. Int. J. Solids Struct. 2007; 44 4925-4941. https://doi.org/10.1016/j.ijsolstr.2006.12.015.

[11] Meade Jr A.J., Fernandez A.A., The numerical solution of linear ordinary differential equations by feedforward neural networks, Mathematical and Computer Modelling. 1994; 19 (12): 1-25. https://doi.org/10.1016/0895-7177(94)90095-7.

[12] Lagaris I.E., Likas A., Fotiadis D.I. Artificial neural networks for solving ordinary and partitial differential equations. IEEE Transactions on Neural Networks. 1998; 9 (5): 987-1000. https://doi.org/10.1109/72.712178.

[13] Malek A., Beidokhti R.S., Numerical solution for high order differential equations using a hybrid neural network-Optimization method. Applied Mathematics and Computation. 2006; 183: 260271. https://doi.org/10.1016/j.amc.2006.05.068.

[14] Lee H., Kang I.S., Neural algorithms for solving differential equations, Journal of Computational Physics 1990; 91: 110-131. https://doi.org/10.1016/0021-9991(90)90007-N.

[15] Yang X.-S., Deb S., "Cuckoo search via L'evy flights”, in: Proc. Of World Congress on Nature \& Biologically Inspired Computing (NaBIC 2009), December 2009, India. IEEE Publications, USA, pp. 210-214 (2009). https://doi.org/10.1109/NABIC.2009.5393690.

[16] Chaowanawatee, K., \& Heednacram, A. (2012, July). Implementation of cuckoo search in RBF neural network for flood forecasting. In Computational Intelligence, Communication Systems and Networks (CICSyN), 2012 Fourth International Conference on (pp. 22 26). IEEE. https://doi.org/10.1109/cicsyn.2012.15.

[17] Santos, C. A., Freire, P. K., \& Mishra, S. K. (2012). Cuckoo search via Lévy flights for optimization of a physically-based runofferosion model. Journal of Urban and Environmental Engineering, 6(2), 123-131. https://doi.org/10.4090/juee.2012.v6n2.123131.

[18] Valian, E., \& Valian, E. (2013). A cuckoo search algorithm by Lévy flights for solving reliability redundancy allocation problems. Engineering Optimization, 45(11), 1273-1286 https://doi.org/10.1080/0305215X.2012.729055.

[19] Kennedy J., Eberhart R.C., Particle swarm optimization. Proc. of IEEE International Conference on Neural Networks, Piscataway, NJ. pp. 1942-1948 (1995). https://doi.org/10.1109/ICNN.1995.488968.

[20] Kennedy J., Eberhart R., Shi Y., Swarm intelligence, Academic Press, (2001).

[21] Ascher U.; Mattheij, R., Russell, R. "Numerical Solution of Boundary Value Problems for Ordinary Differential Equations." SIAM Classics in Applied Mathematics. Vol. 13. (1995). https://doi.org/10.1137/1.9781611971231.

[22] Ascher U., Petzold, L. "Computer Methods for Ordinary Differential Equations and Differential-Algebraic Equations." SIAM, Philadelphia. 1998. https://doi.org/10.1137/1.9781611971392.

[23] Timoshenko S., Theory of Plates and Shells, (New York: McGraw Hill) 1987. 